\title{
The Minimum Numbers for Certain Positive Operators
}

\author{
Ching-Yun Suen
}

Correspondence: Ching-Yun Suen, Foundational Sciences, Texas A\&M University at Galveston, P. O. Box 1675, Galveston, Texas 77553-1675, USA.

Received: June 4, 2020 Accepted: August 5, 2020 Online Published: August 19, 2020

doi:10.5539/jmr.v12n5p15 URL: https://doi.org/10.5539/jmr.v12n5p15

\begin{abstract} for some Hilbert space $H$.

When $T$ is self-adjoint, we have the minimum of $k$.

When $m=3$ and $T \in B(H)$, we obtain the minimum of $k$ and an inequality

Involving the numerical radius $w(T)$.
\end{abstract}

In this paper we give upper and lower bounds of the infimum of $k$ such that $k I+2 \operatorname{Re}\left(T \otimes S_{m}\right)$ is positive, where $S_{m}$ is the $m \times m$ matrix whose entries are all 0's except on the superdiagonal where they are all 1's and $T \in B(H)$

Keywards: positive operators, operator radius, $w_{\rho}$ norms

AMS Subject Classifications (2010): 47A63

\section{Introduction}

Let $B(H)$ denote the algebra of all bounded linear operator on a Hilbert space $H$. Given $T \in B(H)$, we say that $T \in C_{\rho}(0<\rho<\infty)$ if there is a unitary operator $U$ on a Hilbert space $K$ containing $H$ as a subspace, such that $T^{n}=\left.\rho P_{H} U^{n}\right|_{H}(n=1,2,3, \ldots)$. Sz-Nagy and Foias introduced the class $C_{\rho}[6]$, J. A. R. Holbrook[4] and J. P.

Williams[9] defined the operator radii $w_{\rho}(T)=\inf \left\{r>0: \frac{T}{r} \in C_{\rho}\right\}$. When $\rho=2$, then $w_{2}(T)=w(T)$ the numerical radius of $T$. In the paper we provide upper and lower bounds which are best numbers for certain positive operators. Ando and Okubo [1] introduce $D_{\rho}=\left(\begin{array}{cc}0 & \sqrt{\rho(2-\rho)} \\ 0 & 1-\rho\end{array}\right)$ and $\rho w_{\rho}(T)=2 w\left(D_{\rho} \otimes T\right)$ for $1 \leq \rho \leq 2$.

We obtain the minimum of $k$ such that $k I+2 \operatorname{Re}\left(D_{\rho} \otimes T\right) \geq 0$ for all $m \geq 2$ is equal to $\rho w_{\rho}(T)$ for $1 \leq \rho \leq 2$.

If $T$ is self-adjoint, we prove the minimum of $k$ such that

$k I+2 \operatorname{Re}\left(T \otimes S_{m}\right) \geq 0$ for all $m \geq 2$ is equal to $2 \cos \frac{\pi}{m+1}\|T\|$.

Finally, we prove an inequality $\sqrt{2} w(T) \leq\left\|T T^{*}+T^{*} T\right\|^{\frac{1}{2}} \leq 2 w(T)$.

\section{2. $W_{\rho}$ Norms $(1 \leq \rho \leq 2)$}

Definition 2.1. [9] Let $H$ and $K$ be Hilbert spaces and suppose that $U \in B(H)$ and $V \in B(K)$. Then there is a unique operator $U \otimes V \in B(H \otimes K)$ such that

$(U \otimes V)(h \otimes k)=U(h) \otimes V(k)$ for $h \in H$ and $k \in K$.

Lemma 2.2. $w_{\rho}(S \otimes T) \leq\|T\| w_{\rho}(S)$ for $\rho>0$.

Proof. Since $S \otimes I$ and $I \otimes T$ are double commuting operators, applying [5], we have

$$
\begin{gathered}
\left.w_{\rho}(S \otimes T)=w_{\rho}((S \otimes I))(I \otimes T)\right) \\
\leq w_{\rho}(S \otimes I)\|I \otimes T\|=w_{\rho}(S \otimes I)\|T\| .
\end{gathered}
$$


Since $\frac{S}{w_{\rho}(S)}=\left.\rho P_{H} U\right|_{H}$ for some unitary operator $U$ and Hilbert space $H$,

We have

$\left(\frac{S}{w_{\rho}(S)} \otimes I\right)^{n}=\left.\rho\left(P_{H} \otimes I\right)(U \otimes I)^{n}\right|_{H \otimes K}$ for some Hilbert space $K$.

Thus $\frac{s}{w_{\rho}(S)} \otimes I \in C_{\rho}$,

Hence

$w_{\rho}(S \otimes I) \leq w_{\rho}(S)$.

From the proof of [7, Proposition 2.1], we know the following Lemma:

Lemma 2.3. If $\cdots\left(\begin{array}{cccc}2 & T & 0 & 0 \\ T^{*} & 2 & \ddots & 0 \\ 0 & \ddots & \ddots & T \\ 0 & 0 & T^{*} & 2\end{array}\right)_{m \times m} \geq 0$, then $w(T) w\left(S_{m}\right) \leq 1$ where

$S_{m}=\left(\begin{array}{cccc}0 & 1 & 0 & 0 \\ 0 & 0 & \ddots & 0 \\ . & \ddots & \ddots & 1 \\ 0 & . & 0 & 0\end{array}\right)_{m \times m}$ with $m \geq 2$ and $T \in B(H)$.

Proof. Let $\lambda=\left(\begin{array}{c}\lambda_{1} \\ \lambda_{2} \\ \vdots \\ \lambda_{m}\end{array}\right)$ with $\|\lambda\|=1$ and $h_{i}=\lambda_{i} h$ for $i=1,2, \ldots, m$,

where $h \in H$ with $\|h\|=1$, then

$$
\begin{aligned}
& <\left(\begin{array}{cccc}
1 & 0 & \cdots & 0 \\
0 & \bar{z} & \ddots & \vdots \\
\vdots & \ddots & \ddots & 0 \\
0 & \cdots & 0 & \bar{z}^{m-1}
\end{array}\right)\left(\begin{array}{cccc}
2 & T & 0 & 0 \\
T^{*} & 2 & \ddots & 0 \\
0 & \ddots & \ddots & T \\
0 & 0 & T^{*} & 2
\end{array}\right)_{m \times m}\left(\begin{array}{cccc}
1 & 0 & \cdots & 0 \\
0 & z & \ddots & \vdots \\
\vdots & \ddots & \ddots & 0 \\
0 & \cdots & 0 & \bar{z}^{m-1}
\end{array}\right)\left(\begin{array}{c}
h_{1} \\
h_{2} \\
\vdots \\
h_{m}
\end{array}\right), \\
& \left(\begin{array}{c}
h_{1} \\
h_{2} \\
\vdots \\
h_{m}
\end{array}\right)>=<\left(\begin{array}{cccc}
2 & z T & 0 & 0 \\
\bar{z} T^{*} & 2 & \ddots & 0 \\
0 & \ddots & \ddots & z T \\
0 & 0 & \bar{z} T^{*} & 2
\end{array}\right)_{m \times m}\left(\begin{array}{c}
h_{1} \\
h_{2} \\
\vdots \\
h_{m}
\end{array}\right),\left(\begin{array}{c}
h_{1} \\
h_{2} \\
\vdots \\
h_{m}
\end{array}\right)> \\
& =2+2 \operatorname{Re}\left(z<S_{m} \lambda, \lambda><T h, h>\right) \geq 0 \text { for }|z|=1 .
\end{aligned}
$$

Similarly, we have

$2-2 \operatorname{Re}\left(z<S_{m} \lambda, \lambda><T h, h>\right) \geq 0$

Thus

$\left\lceil\operatorname{Rez}<S_{m} \lambda, \lambda><T h, h>\mid \leq 1\right.$ for all $|z|=1$.

Hence

$\left|<S_{m} \lambda, \lambda><T h, h>\right| \leq 1$.

From [1], we know that $D_{\rho}=\left(\begin{array}{cc}0 & \sqrt{\rho(2-\rho)} \\ 0 & 1-\rho\end{array}\right)$ and $\rho w_{\rho}(T)=2 w\left(D_{\rho} \otimes T\right)$ for $1 \leq \rho \leq 2$.

Lemma 2.4 .

$w\left(S_{m}\right) \leq w_{\rho}\left(S_{m}\right) \leq \frac{2}{\rho} w\left(S_{m}\right)$, for $1 \leq \rho \leq 2$ and $m \geq 2$.

Proof. Applying Lemma 2.2, we have $w_{\rho}\left(S_{m}\right)=\frac{2}{\rho} w\left(D_{\rho} \otimes S_{m}\right) \leq \frac{2}{\rho} w\left(S_{m}\right)$.

We have upper and lower bounds for certain positive operators in the following: 
Theorem 2.5. $2 w\left(S_{m}\right) w(T) \leq$ inf $\left\{\left\{k:\left(\begin{array}{cccc}k & T & 0 & 0 \\ T^{*} & k & \ddots & 0 \\ 0 & \ddots & \ddots & T \\ 0 & 0 & T^{*} & k\end{array}\right)_{m \times m} \geq 0\right\}\right.$

$\leq 2 w\left(S_{m}\right)\|T\|$ for a fixed positive integer $m \geq 2$.

Proof. Applying Lemma 2.3 , if $\cdot\left(\begin{array}{cccc}k & T & 0 & 0 \\ T^{*} & k & \ddots & 0 \\ 0 & \ddots & \ddots & T \\ 0 & 0 & T^{*} & k\end{array}\right)_{m \times m} \geq 0$

then

$2 w(T) w\left(S_{m}\right) \leq k$.

Applying Lemma 2.2, we have

$$
w\left(S_{m}\right)\|T\| \geq w\left(T \otimes S_{m}\right)=\sup _{|\lambda|=1}\left\{\operatorname{Re} \lambda\left(T \otimes S_{m}\right)\right.
$$

$\geq \operatorname{Re} \lambda\left(T \otimes S_{m}\right)$, for all $|\lambda|=1$.

We have the $m \times m$ matrix $\left(\begin{array}{ccccc}2 w\left(S_{m}\right)\|T\| & T & & 0 & 0 \\ T^{*} & \ddots & \ddots & 0 \\ 0 & \ddots & \ddots & T \\ 0 & 0 & T^{*} & 2 w\left(S_{m}\right)\|T\|\end{array}\right)$

$=2 w\left(S_{m}\right)\|T\|+T \otimes S_{m}+T^{*} \otimes S_{m}{ }^{*}=2\left(w\left(S_{m}\right)\|T\|+\operatorname{Re}\left(T \otimes S_{m}\right)\right) \geq 0$.

If $T$ is self-adjoint, we obtain the minimum value of $k$ in the following:

Corollary 2.6. If $T=T^{*}$, then

$\min \left\{k:\left(\begin{array}{cccc}k & T & 0 & 0 \\ T^{*} & k & \ddots & 0 \\ 0 & \ddots & \ddots & T \\ 0 & 0 & T^{*} & k\end{array}\right)_{m \times m} \geq 0\right\}=2 w\left(S_{m}\right)\|T\|=2 \cos \frac{\pi}{m+1}\|T\|$, for $m \geq 2$.

Proof. From [3], we know that $\left(S_{m}\right)=\cos \frac{\pi}{m+1}$.

Applying Lemma 2.2 and Theorem 2.5, we have the following Theorem:

\section{Theorem 2.7 .}

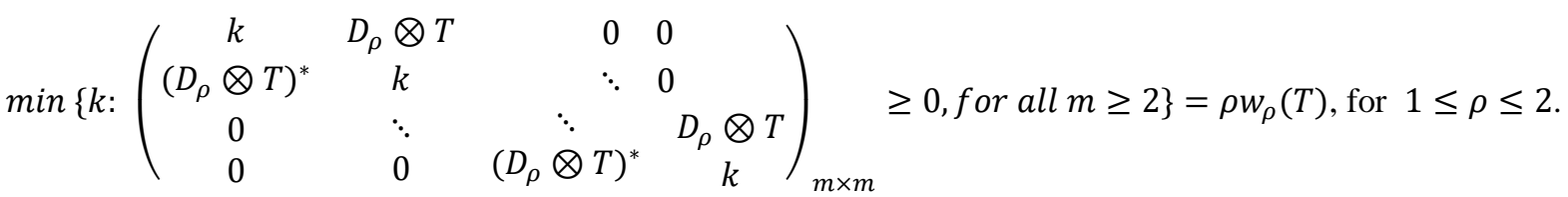

Proof. Applying Lemma 2.2, we have $w\left(D_{\rho} \otimes T\right) \geq w\left(D_{\rho} \otimes T \otimes S_{m}\right)$ for all $m \geq 2$. From the proof of Theorem 2.5, we also have the $m \times m$ matrix

$$
\begin{gathered}
\left(\begin{array}{cccc}
2 w\left(D_{\rho} \otimes T\right) & D_{\rho} \otimes T & 0 & 0 \\
\left(D_{\rho} \otimes T\right)^{*} & \ddots & \ddots & 0 \\
0 & \ddots & \ddots & D_{\rho} \otimes T \\
0 & 0 & \left(D_{\rho} \otimes T\right)^{*} & 2 w\left(D_{\rho} \otimes T\right)
\end{array}\right) \\
=2\left(w\left(D_{\rho} \otimes T\right)+\operatorname{Re}\left(D_{\rho} \otimes T \otimes S_{m}\right)\right) \geq 0 .
\end{gathered}
$$

Applying Theorem 2.5, we have

$$
2 w\left(S_{m}\right) w\left(D_{\rho} \otimes T\right) \leq
$$


$\inf \left\{k:\left(\begin{array}{cccc}k & D_{\rho} \otimes T & & 0 \\ \left(D_{\rho} \otimes T\right)^{*} & k & \ddots & 0 \\ 0 & \ddots & \ddots & D_{\rho} \otimes T \\ 0 & 0 & \left(D_{\rho} \otimes T\right)^{*} & k\end{array}\right)_{m \times m} \geq 0\right\}$

$\leq 2 \mathrm{w}\left(D_{\rho} \otimes T\right)=\rho w_{\rho}(T)$, for all $m \geq 2$ and $1 \leq \rho \leq 2$.

Let $m \rightarrow \infty$, we have the Theorem.

We obtain [7, Proposition 2.2] in the following:

Corollary 2.8. $\min \left\{k:\left(\begin{array}{cccc}k & T & 0 & 0 \\ T^{*} & k & \ddots & 0 \\ 0 & \ddots & \ddots & T \\ 0 & 0 & T^{*} & k\end{array}\right)_{m \times m} \geq 0\right.$, for all $\left.m \geq 2\right\}=2 w(T)$.

Proof. Let $\rho=2$ in Theorem 2.7.

Example 2.9. From Corollary 2.6, we have

$$
\min \left\{k:\left(\begin{array}{cccc}
k & 1 & 0 & 0 \\
1 & k & \ddots & 0 \\
0 & \ddots & \ddots & 1 \\
0 & 0 & 1 & k
\end{array}\right)_{m \times m} \geq 0, \text { for } m \geq 2\right\}=2 \cos \frac{\pi}{m+1}
$$

and from Corollary 2.8, we have

$$
\min \left\{k:\left(\begin{array}{cccc}
k & 1 & 0 & 0 \\
1 & k & \ddots & 0 \\
0 & \ddots & \ddots & 1 \\
0 & 0 & 1 & k
\end{array}\right)_{m \times m} \geq 0, \text { for all } m \geq 2\right\}=2
$$

Corollary 2.10. If $T$ is idempotent (that is, $T^{2}=T$ ) and $T \neq 0$,

Then $\min \left\{k:\left(\begin{array}{cccc}k & D_{\rho} \otimes T & 0 & 0 \\ \left(D_{\rho} \otimes T\right)^{*} & k & \ddots & 0 \\ 0 & \ddots & \ddots & D_{\rho} \otimes T \\ 0 & 0 & \left(D_{\rho} \otimes T\right)^{*} & k\end{array}\right)_{m \times m} \geq 0\right.$, for all $\left.m \geq 2\right\}=\|T\|+\rho-1$ for $1 \leq \rho \leq 2$.

Proof. By [2, Theorem 6(1)], $\rho w_{\rho}(T)=\|T\|+\rho-1$.

We obtain an inequality involving the numerical radius of $T$ in the following:

Corollary 2.11. $\sqrt{2} w(T) \leq\left\|T T^{*}+T^{*} T\right\|^{\frac{1}{2}} \leq 2 w(T)$. Moreover, if $T$ is normal (that is $T^{*} T=T T^{*}$ ), then $\|T\| \leq \sqrt{2} w(T)$.

Proof. From [8], we have in $\left\{k:\left(\begin{array}{ccc}k & T & 0 \\ T^{*} & k & T \\ 0 & T^{*} & k\end{array}\right) \geq 0\right\}=\left\|T T^{*}+T^{*} T\right\|^{\frac{1}{2}}$.

By Theorem 2.5 and Corollary 2.8, we have

$2 w\left(S_{3}\right) w(T) \leq\left\|T T^{*}+T^{*} T\right\|^{\frac{1}{2}} \leq 2 w(T)$.

Example 2.12. Since $2 w\left(s_{3}\right) w\left(s_{2}\right)=\frac{\sqrt{2}}{2} \leq \min \left\{k:\left(\begin{array}{ccc}k & s_{2} & 0 \\ s_{2}{ }^{*} & k & s_{2} \\ 0 & s_{2}{ }^{*} & k\end{array}\right) \geq 0\right\}=1 \leq 2 w\left(s_{3}\right)\left\|s_{2}\right\|=\sqrt{2}$, we have the lower and upper bounds of Theorem 2.5. Also, $\sqrt{2} w(T)$ and $2 w(T)$ are the best constants in the inequality of Corollary 2.11 .

\section{Conclusion}

We have minimum norms for certain positive operators with finite or infinite size. 


\section{References}

Ando, T., \& Nishio, K. (1973). Convexity properties of operator radii associated with $\rho$-dilations. Michigan Math. J., 20, 303-307. https://doi.org/10.1307/mmj/1029001147

Ando, T., \& Okubo, K. (1991). Induced norms of the Schur multiplier operator, Linear Algebra Appl., 147, $181-199$. https://doi.org/10.1016/0024-3795(91)90234-N

Haagerup, U., \& de la Harpe, P. (1992). The numerical radius of a nilpotent operator on a Hilbert space. Proc. Amer. Math, 115, 371-379. https://doi.org/10.1090/S0002-9939-1992-1072339-6

Holbrook, J. A. R. (1968). On the power-bounded operators of Sz-Nagy and Foias. Acta Sci. Math. (Szeged), 29, 299-310. https://doi.org/10.1307/mmj/1029000638

Holbrook, J. A. R. (1971). Inequalities governing the operator radii associated with unitary $\rho$-dilations. Michigan Math. J., $18,149-159$.

Suen, C. Y. (1995). The minimum norm of certain completely positive maps, Proc. Amer. Math., 123, $2407-2416$. https://doi.org/10.1090/S0002-9939-1995-1213870-2

Suen, C. Y. (2018). Strictly positivity of operators and inflated Schur product. J. of Math. Research, 10, $30-42$. https://doi.org/10.5539/jmr.v10n6p30

Sz-Nagy, B., \& Foias, C. (1996). On certain classes of power-bounded operators in Hilbert space. Acta Sci. Math. (Szeged), 27, 17-25.

Takesaki, M. (1979). Theory of operator algebra I, Springer. Berlin, Heidelberg, and New York. https://doi.org/10.1007/978-1-4612-6188-9

Williams, J. P. (1968). Schwarz norms for operators. Pacific J. Math., 24, 181-188. https://doi.org/10.2140/pjm.1968.24.181

\section{Copyrights}

Copyright for this article is retained by the author(s), with first publication rights granted to the journal.

This is an open-access article distributed under the terms and conditions of the Creative Commons Attribution license (http://creativecommons.org/licenses/by/4.0/). 